\title{
Calcitonina monomérica plasmática e hipercalcemia em pacientes portadores de neoplasia pulmonar
}

\author{
R. Coifman, R.V.B. Weforte, T.S. Kasamatsu, L. Fukusima, I. Santoro, S. Jamnik, J .G.h. Vieira, \\ M.L. CASTRO \\ Disciplina de Endocrinologia, Universidade Federal de São Paulo - Escola Paulista de Medicina, São Paulo, SP.
}

\begin{abstract}
RESUMO - A calcitonina (CT) é um hormônio peptídico relacionado ao metabolismo de cálcio produzido pelas células $C$ da tiróide. Encontra-se com níveis plasmáticos bastante elevados no carcinoma medular de ti róide e mostra-se como excelente marcador dessa doença. No entanto, existem relatos na literatura que demonstraram níveis elevados deste peptídio em pacientes portadores de outras neoplasias, principalmente no carcinoma de pulmão.

Oвj eтıvo. Avaliar a validade da dosagem da CT sérica como possível marcador tumoral em pacientes portadores de tumor de pulmão de diferentes tipos histológicos.

MÉtodos. Foram dosados CT plasmática e cálcio ionizado sanguíneo em 56 pacientes portadores de tumores malignos de pulmão. Para as dosagens de CT os autores utilizaram um método de radio-
\end{abstract}

\section{NTRODUÇÃO}

A neoplasia de pulmão é a maior causa de morte por câncer em homens e é a segunda maior causa de óbito por câncer nas mulheres, após o câncer de mama ${ }^{1}$. Devido a esta al ta inci dência, é constante a busca por marcadores tumorais eficientes, não apenas para detecção precoce da malignidade, mas também para a avaliação de prognóstico e acompanhamento após o tratamento cirúrgico, radioterápico ou clínico dos tumores de pulmão.

Os marcadores tumorais disponíveis mais utilizados são o antígeno carcinoembrionário (CEA) e a al fa-fetoproteína, com resultados que deixam muito a desejar em questão de sensibilidade e especificidade $^{2-4}$. Muitos dos tumores pulmonares estão associados a secreções paraneoplásicas de hormônios peptídicos, que podem, até mesmo, levar a um quadro clínico específico, como a síndrome de Cushing nos tumores de pequenas células produtores de ACTH, ou a hipercalcemia associada à malignidade, nos casos de secreção de PTH-related protein (PTHrP) nos carcinomas espinoce-lulares. Nestes casos, pode-se utilizar as concentrações plamáticas destes hormônios como marcadores tumorai s $^{5,6}$.

A calcitonina, um outro hormônio polipeptídico secretado principalmente pelas células C da ti rói- imunoensaio específico, realizado após extração prévia do soro em coluna de sílica.

Resultados. Observou-se prevalência de hipercalcemia de $21,4 \%$; apenas três $(5,4 \%)$ dos 56 pacientes investigados apresentaram níveis pouco elevados de calcitonina, e o restante manteve níveis normais ou indetectáveis do peptídio.

Conclusão. Os resultados demonstram que, com a utilização de um método bastante específico para dosagem da calcitonina em sua forma monomérica, não se encontram níveis elevados deste hormônio em pacientes portadores de neoplasia pulmonar, desestimulando sua utilização como marcador tumoral nesta patologia.

UNITERMOS: Calcitonina. Neoplasia pulmonar. Marcador tumoral. Hipercalcemia.

de, está relacionada à manutenção dos níveis normais de cál cio plasmáti co e à proteção do osso contra a desmineralização. El evações agudas dos níveis de cálcio plasmático estimulam a secreção de calcitonina, que, por sua vez, exerce seu efeito hipocalcemiante inibindo a ação dos osteoclastos na reabsorção óssea e retardando a absorção intestinal de cál cio9. Sua presença em níveis bastante elevados é observada no sangue de pacientes portadores de carcinoma medular de tiróide. Neste caso, a CT é um excel ente marcador tumoral, utilizado não somente para diagnóstico mas, também, como parâmetro de evolução e eficácia do tratamento9. Excluindo-se o carcinoma medular de tiróide, a presença de níveis el evados de CT foi descrita por alguns autores no plasma de pacientes portadores de outras neoplasias malignas, principalmente nos carcinomas pulmonares. São estudos relativamente antigos, que remontam à época de desenvolvimento dos primeiros radioimunoensaios para determi nação de $\mathrm{CT}$. No entanto, a partir destas descrições, a indicação da utilização da CT como marcador tumoral é constantemente citada em diversos trabalhos, até mesmo em livros textos clássicos de medicina ${ }^{10-13}$. Estudos in vitro mais recentes demonstraram a capacidade de algumas linhagens celulares de 
tumores de pulmão e próstata ${ }^{12,14-17}$ de secretar calcitonina, além de outros hormônios polipeptídicos como ACTH, CGRP (cal citonin generelated peptide) e PTHrP.

Tendo em nossas mãos um ensaio bastante sensível e específico para dosar CT, somado a um bem estruturado ambulatório de oncopneumologia, resolvemos avaliar a utilidade das dosagems de CT plasmáticas como marcador tumoral em pacientes portadores de neoplasia maligna de pulmão que freqüentaram esse ambulatório.

\section{MATERIAL E MÉTODOS}

F oram estudados 56 pacientes de forma consecutiva, que freqüentaram o Ambulatório de Oncopneumologia da Disciplina de Pneumologia da Escola Paulista de Medicina, no período de abril a outubro de 1992. Todos apresentavam diagnóstico comprovado de neoplasia maligna pulmonar, em diferentes graus de estadiamento e submetidos a diferentes terapêuticas. Eram 38 homens e 18 mulheres, com idade média de 61,4 anos (35 a 87 anos), portadores dos seguintes tipos histológicos: $16(28,5 \%)$ pacientes com carcinoma espinocelular (CEC); 24 (42,8\%) pacientes com adenocarcinoma; $12(21,4 \%)$ pacientes com carcinoma indiferenciado de pequenas células (CIPC) ; três $(5,3 \%)$ pacientes com carcinoma indiferenciado $(\mathrm{Cl})$ e um $(1,8 \%)$ paciente com blastoma. Em todos os pacientes foram dosados $\mathrm{Ca}$ ionizado (Cai) em sangue total e calcitonina sérica (CT). Os níveis de paratormônio (PTH) foram determinados nos pacientes que se apresentavam hipercal cêmi cos na ocasião da coleta, com o objetivo de afastar um possível hiperparatiroidismo primário. Em 30 pacientes, a presença ou não de metástase óssea foi determinada por meio de mapeamento ósseo com tecnécio (99mTc). O Cai foi dosado por meio do método el etrodo íon-específico (Radi ometer, ICA-2, Dinamarca), e o PTH aminoterminal por radioimunoensaio já descrito anteri ormente ${ }^{18,19}$.

$O$ radioimunoensaio para dosagem de CT baseia-se no método descrito por Body et al. ${ }^{20}$, e utiliza anti-soro produzido em coel hos contra CT humana sintética (Bachen Fine Chemicals, EUA), empregada no ensaio a uma diluição final de 1:40.000, que não apresenta reação cruzada com insulina, ACTH, gastrina, pentagastrina, colecistoquinina, PTH bovino ou humano. A mesma CT humana sintética foi utilizada como padrão para marcação com ${ }^{125}$ | (Amershan). A marcação com ${ }^{125}$ | empregou a técnica da Cloramina T (Merck), seguida de purificação em sistema de cromatografia líquida de alta performance (HLPC - Millipore).
Tabela - Dados clínicos e laboratoriais dos três pacientes portadores de neoplasia pulmonar com níveis de CT elevados

\begin{tabular}{|lcccc|}
\hline Sexo/Idade & $\begin{array}{c}\text { Tipo } \\
\text { histológico }\end{array}$ & $\begin{array}{c}\text { Cai } \\
(\mathbf{m M})\end{array}$ & $\begin{array}{c}\mathbf{C T} \\
\text { (pmol/L) }\end{array}$ & $\begin{array}{c}\text { Metástase } \\
\text { óssea }\end{array}$ \\
masc/72a & CIPC & 1,25 & 36 & ausente \\
fem/49a & Adeno & 1,25 & 20 & presente \\
masc/49a & CEC & 1,27 & 19,1 & ausente \\
\hline
\end{tabular}

CIPC: carcinoma indiferenciado de pequenas células. Adeno: adenocarcinoma. CEC: carcinoma espinocelular. Valores normais: Cai - 1,13 $1,29 \mathrm{mmol} / \mathrm{L} ; \mathrm{CT}$ - até $18 \mathrm{pmol} / \mathrm{L}$.

Dois mililitros de soro das amostras eram extraídos previamente em coluna de Seppak C 18 (Millipore), com posterior reconstituição em $0,5 \mathrm{~mL}$ de tampão do ensaio. Em seguida, as amostras extraídas eram incubadas com o anti-soro por 48 horas, quando então a CT marcada com ${ }^{125}$ | era adicionada, seguidas de mais 48 horas de incubação. A separação era feita com o emprego de um segundo anticorpo anti-I gG de coel ho produzido em carneiro. Após uma hora, polietilenoglicol (PEG - Merck) $6,4 \%$ era adicionado, as amostras centrifugadas por 30 minutos a $3.000 \mathrm{rpm}$. O sobrenadante era decantado, e o precipitado medido em contador gama por um minuto. O limite de detecção do ensaio é da ordem de $1 \mathrm{pmol} / \mathrm{L}$. Todos os ensaios foram feitos em duplicata, considerando-se como normais valores de até $18 \mathrm{pmol} / \mathrm{L}$. A CT sérica também foi dosada em três indivíduos portadores de carcinoma medular de tiróide.

\section{RESULTADOS}

Foram encontrados níveis normais ou indetectáveis de calcitonina em 53 indivíduos $(94,6 \%)$ portadores de neoplasia pulmonar. Em apenas três pacientes $(5,4 \%)$ esses níveis ultrapassaram o limite superior da normalidade, e em dois deles os valores estavam apenas discretamente el evados (tabela).

Hipercalcemia (Cai $>1,29 \mathrm{mmol} / \mathrm{L}$ ) foi detectada em 12 pacientes $(21,4 \%)$ : cinco portadores de CIPC $(41,6 \%)$, cinco de CEC (41,6\%) e dois de adenocarcinoma (16,5\%). Todos os pacientes hipercalcêmicos mantiveram níveis de PTH sérico suprimidos ou normais, afastando o diagnóstico de hiperparatiroidismo primário. Apesar da hipercalcemia, nenhum destes pacientes apresentou níveis elevados de calcitonina, e em cinco $(41,7 \%)$ os valores de calcitonina estavam indetectáveis. Todos os três pacientes com níveis el evados de CT apresentavam-se normocal cêmicos (fig. 1). Dezoito (60\%) dos 30 pacientes submetidos à investigação de metástase óssea apresentaram mapeamento ósseo com 


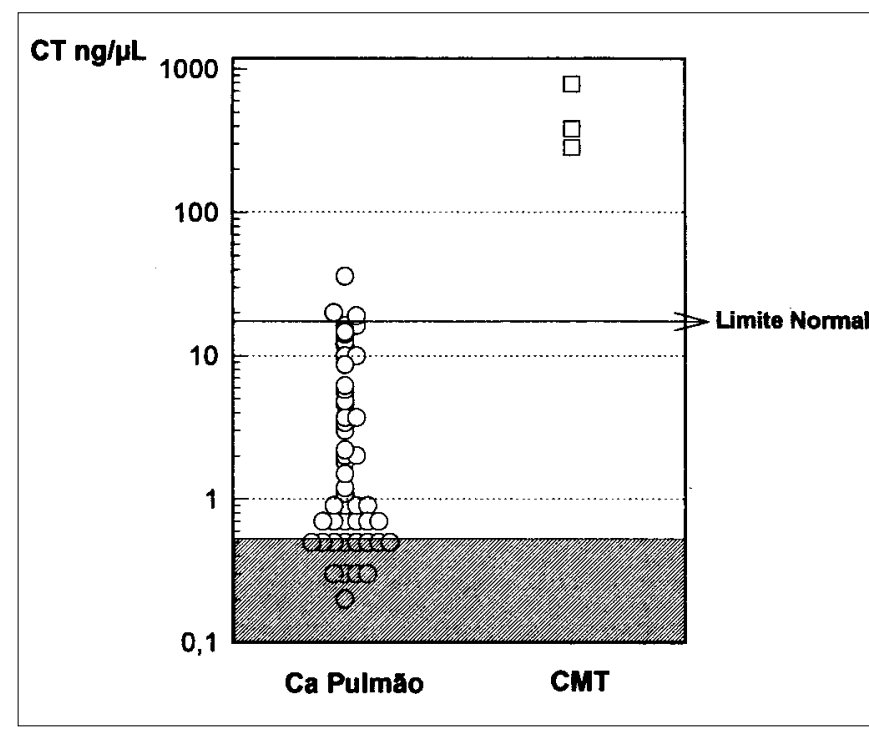

Fig. - Níveis plasmáticos de calcitonina monomérica em pacientes portadores de câncer de pulmão (Ca pulmão) e carcinoma medular de ti róide (CMT).

concentração anômal a do radioindicador, e apenas um destes possuía níveis de calcitonina discretamente elevados. Os pacientes portadores de carcinoma medular de tiróide apresentaram níveis el evados de CT, acima de 200pmol/L (figura).

\section{DISCUSSÃO}

A CT é secretada pelas células $C$ da tiróide, e utilizada como um eficiente marcador tumoral no diagnóstico e seguimento do carcinoma medular da tiróide. $\mathrm{Na}$ década de 70 diversos autores relataram casos de aumento da secreção deste peptídio em portadores de neoplasia mali gna pulmonar, em $50 \%$ a $60 \%$ dos $\operatorname{casos}^{11,13-15}$. Nesses mesmos estudos demonstrou-se que os níveis da calcitonina diminuíram após o tratamento antitumoral específico, fazendo com que os autores preconizassem o uso desse hormônio como marcador tumoral no diagnóstico e prognóstico desses tumores. Nossos resultados contrapõem-se a esses achados, pois encontramos níveis el evados de CT em apenas três $(5,4 \%)$ dos 56 pacientes investigados, desestimulando o uso da CT como marcador tumoral. Os níveis encontravam-se apenas discretamente elevados, contrastando com os obtidos nos pacientes portadores de carcinoma medular de tiróide. Como uma parte desses pacientes estava em vigência de algum tipo de terapêutica antitumoral, poderíamos pensar que estivessem em remissão e, devido a isso, apresentassem níveis normais de CT plasmática. Doze (21,4\%) desses pacientes, no entanto, apresentavam-se hipercal cêmicos no momento do estudo, indicando doença em plena atividade ${ }^{21,22}$. Apesar disso, em nenhum deles foram constatados níveis el evados de calcitonina.

U ma correlação entre níveis el evados de CT e a presença de metástases ósseas foi observada em um dos trabalhos citados ${ }^{23}$; entretanto, encontramos CT el evada em apenas um dos 18 pacientes portadores de metástases ósseas por nós avaliados.

Acreditamos que esses resultados conflitantes se devam, principalmente, ao método de dosagem de CT utilizados nos diferentes estudos. A forma monomérica da calcitonina é constituída por apenas 32 aminoácidos e apresenta níveis circulantes baixos, na ordem de alguns picomoles/ $\mathrm{L}^{9}$. Os estudos anteriores nunca atingiam essa faixa, e seus níveis normais eram muito superiores a estes ${ }^{13-15}$. A grande maioria dos indivíduos normais apresentava níveis indetectáveis, mostrando que a sensibilidade desses métodos deixava muito a desejar. A constatação da existência de formas heterogêneas deCT circulantes, reconhecidas indiscriminadamente por diversos anti-soros utilizados nesses radioimunoensaios, comprovou serem esses métodos também inespecífi $\cos ^{24,25}$. A utilização de extração prévia do soro possibilitou uma melhora sensível dos ensaios, concentrando a amostra e isolando apenas a forma monomérica da $\mathrm{CT}$, considerada a biologicamente ativa. O método utilizado por nós apresenta exatamente estas características: utiliza extração prévia e um anti-soro al tamentesensível eespecífico, chegando muito próximo à faixa de normalidade. Portanto, acreditamos que esses achados antigos traduzem, provavel mente, a inespecificidade dos métodos utilizados, eque os níveis el evados encontrados por esses autores não sejam exclusivamente de CT, mas de uma série de outros peptídios, fragmentos de molécula ou precursores que pudessem estar sendo produzidos por esses tumores ${ }^{24,25}$.

Nossos dados salientam, também, um outro aspecto interessante. Como a CT é um hormônio liberado quando ocorre aumento dos níveis de Ca no plasma, seria de se esperar níveis el evados desse peptídio nos pacientes que se encontravam hipercal cêmicos. Mas, ao contrário, todos apresentavam-se com níveis normais ou indosáveis de CT, confirmando, assim, a perda da resposta das células $C$ à hipercalcemia crônica já descrita por outros pesquisadores ${ }^{26,27}$. Estudos in vitro demonstram haver uma diminuição da sensibilidade das células $C$ a níveis suprafisiológicos de $\mathrm{Ca}$, após exposição prolongada ${ }^{28}$.

Concluindo, os resultados obtidos nesse trabaIho demonstram que a $\mathrm{CT}$ plasmática monomérica não é um marcador tumoral útil no acompanhamento dos tumores de pulmão, como os ensaios inespecíficos utilizados em estudos anteriores. 


\section{SUMMARY}

\section{Monomeric plasmatic calcitonin and hyper- calcemia in lung cancer patients}

BACKGRound. Calcitonin (CT) is a peptidic hormone produced by the thyroid $\mathrm{C}$ cells and related to calcium metabolism. High plasmatic levels of this hormone are found in patients with medullary thyroid carcinoma, what makes it an excellent tumor marker for this disease. However, there are reports that showed an increase of plasmatic CT levels in patients with other tumors, mainly in lung cancer.

PURPOSE. These data prompt us to investigate the validity of the CT level determinations as a potential tumor marker in different histologic lung cancer, and its correlation with hypercalcemia, a very common complication in these tumors.

Method. Blood were sampled from 56 patients with malignant lung disease for the $\mathrm{CT}$ and ionized calcium determinations. Calcitonin was measured using a specific radioimmunoassay for the monomeric form of the molecule, in a previous silica extracted serum probe.

RESULTS. Wedid not find el evated level s of monomeric CT in lung cancer. Only 3 patients had mild el evated levels, while in the others $C T$ was normal or undetectable. Hypercalcemia was found in $21.4 \%$ of these patients, but only one with supranormal CT levels.

ConcLusion. Monomeric CT serum levels are normal in lung cancer, what makes the latter use an unreliable tumor marker. [Rev Ass Med Brasil 1997; 43(2): 105-8.]

KEY WORDS: Calcitonin. Lung cancer. Tumor marker. Hypercalcemia.

\section{REFERÊ NCIAS BIBLIOGRÁFICAS}

1. Wittman C, Binderman I. Lung cancer: to operate or not? Am Rev Respir Dis 1977; 116: 477-89.

2. Zamchick N. The present status of CEA in diagnoses, detection of recurrence, prognoses and evaluation of therapy of colonic and pancreatic cancer. Clin Gastroenterol 1976; 5: 625-37.

3. Waldmann TA, Mc Intire KR. The use of radioimmunoassay for al pha-feto protein in the diagnoses of malignancy. Cancer 1974; 34: 1.510-6.

4. Rosen SW, Weintraub BD, Vaitukaitus J L, Sussman $\mathrm{HH}$, Hershman J M, Mugia FM. Placental proteins and their subunits as tumor markers. Ann Intern Med 1975; 82: 71-6.

5. Gewitz G, Yalow RS. Ectopic ACTH production in carcinoma of the lung. J Clin Invest 1974; 53: 1.022-8.

6. Wolfsen AR, Odell WD. Pro ACTH use for early detection of lung cancer. Am J Med 1979; 66: 765-9.

7. Stewler GJ , Nissenson RA. Non parathyroid hypercalcemia.
Adv Intern Med 1987; 32: 235-59.

8. $\mathrm{Ng} \mathrm{KW}$, Martin TJ . Humoral hypercalcemia of malignancy. Clin Biochemistry 1990; 23: 11-25.

9. Raue F. Recent results in cancer research. Medullary thyroid carcinoma. Heildeberg, Springer-Verlag, 1992; 1-89.

10. Odell WD. Humoral manifestations of cancer. In: Willians textbook of endocrinol ogy, F oster W (ed), WB Saunders, Philadel phia, 1985; 1.338-9.

11. Combes RC, Hillyard C, Grenberg PB, Mc Intyre I. Plasma immunoreactive calcitonin in patients with non thyroid tumors. Lancet 1974; 1: 1.080-3.

12. Shulkes A, Fletcher DR, Rubinstin C, Ebeling PR, Martin TJ . Production of calcitonin gene related peptide, calcitonin and PTH rp by a prostatic adenocarcinoma. Clin Endocrinol 1991; 34: 387-93.

13. Silva OL, Becker KL, Primack A, Doppman J L, Snider RH. Ectopic secretion of calcitonin by oat-cell carcinoma. $\mathrm{N}$ Engl J Med 1974; 16: 1.122-3.

14. Silva OL, Becker KL, Primack A, Doppman J L, Snider RH. Increased serum calcitonin levels in bronchogenic cancer. Chest 1976; 69(4): 495-9.

15. Silva O.L, Broder LE, Snider RH, Moore C, Cohen M M, becker $\mathrm{KL}$. Calcitonin as a marker for bronchogenic cancer. Cancer 1979; 44: 680-4.

16. Martin E ME, Gould VE, Rosen ST, Radosevich J A, Deftos LJ . PTHrp, chromogranin A, and calcitonin gene products in the neuroendocrine skin carcinoma cell lives MKL1 and MKL2. Bone Mineral 1991; 14: 113-20.

17. Gerbaud P, Segond N, M onkthar MM, Evain-Brion D. Calcitonin and CGRP chemotatic for F 9 embryonal carcinoma cells. Endocrinology 1991; 1.229(5): 2.530-4.

18. Vieira J GH, Oliveira MAD, RussoEMK, Maciel RMB, Pereira $A B$. E gg yolk as a source of antibodies for human parathyroid hormone radioimmunoassay. J I mmunoassay 1984; 5: 121-6.

19. Vieira J GH, Keutmann FHT, Neer RM. Characterization of a high affinity antiserum specific for the amino-terminal sequence of hPTH. Braz J Med Biol Res 1987; 20: 721-6.

20. Body J J , Heath III. E stimates of circulating monomeric calcitonin: physiological studies in normal and thyroidectomized man. J Clin Endocrinol Metab 1983; 57: 897-903.

21. Bajuronas DR, Muggia FM, Mundy GR, Singer FR. Advances in the hypercalcemia of malignancy. Semin Oncol 1990; 17(2,5): 3-25

22. KaoPC, Klee GG, Taylor RL, Heath III I. PTHrp in plasma of patients with hypercalcemia and malignant lesions. Mayo Clin Proc 1990; 65: 1.399-418.

23. Dambacher MA, Hunziker W, Fisher J A. Die beteutung des plasma-calcitonins fur die klinishe diagnostik. Aktuelle Diagnostik 1977; 33(19): 1.191-3.

24. Singer FR, Habener J F. Multiple immunreactive forms of calcitonin in human plasma. Biochem Biophys Res Commun 1974; 61(2): 660-6.

25. Deftos LJ , Roos BA, Bronzert D, Parthemore J G. Immunochemical heterogenety of calcitonin in plasma. J Clin Endocrinol Metab 1975; 40: 409-12.

26. RaueF, DeutschleL, Kuortzel C, Ziegler R. Reversiblediminished calcitonin secretion in the rat during chronic hypercalcemia. Endocrinol ogy 1984; 154: 2.362-7.

27. Tiegs RD, Body J , BartaJ M, Heath III I. Plasma calcitonin in primary hiperparathyroidism: failure of C-cell response to sustained hypercalcemia. J Clin Endocrinol Metab 1986; 63: 785-92.

28. Mekonnen Y, Raue F, Ziegler R. In vitro secretion of calcitonin from a rat $C$ cell line: effect of a repetitive stimulation with the calcium channel agonist Bay K 8644. Horm Metab Res 1992; 24: 272-5. 